BULLETIN OF THE

AMERICAN MATHEMATICAL SOCIETY

Volume 79, Number 1, January 1973

\title{
FINITELY GENERATED SUBMODULES OF DIFFERENTIABLE FUNCTIONS. II
}

\author{
BY B. ROTH
}

Communicated by François Treves, July 24, 1972

1. Introduction. Let $\mathscr{E}(\Omega)$ denote the space of real-valued infinitely differentiable functions on an open set $\Omega$ in $\mathscr{R}^{n}$ equipped with the topology of uniform convergence of all derivatives on all compact subsets of $\Omega$. Throughout we assume that $\Omega$ is connected.

Let $[\mathscr{E}(\Omega)]^{p}$ denote the Cartesian product of $\mathscr{E}(\Omega)$ with itself $p$-times equipped with the product topology. Then $[\mathscr{E}(\Omega)]^{p}$ is a Frechet space and a $\mathscr{E}(\Omega)$-module. In [3], the finitely generated submodules of $\left[\mathscr{E}^{m}(\Omega)\right]^{p}$ which are closed in $\left[\mathscr{E}^{m}(\Omega)\right]^{p}$ are characterized for $m<\infty$ and we are here concerned with the same problem for $m=\infty$.

2. The main result. Consider the finitely generated submodule $M=$ $\left\{g_{1} f_{1}+\cdots+g_{q} f_{q}: g_{1}, \ldots, g_{q} \in \mathscr{E}(\Omega)\right\}$ of $[\mathscr{E}(\Omega)]^{p}$ where $f_{j}=\left(f_{1 j}, \ldots, f_{p j}\right) \in$ $[\mathscr{E}(\Omega)]^{p}$ for $1 \leqq j \leqq q$. Let $F$ be the $p \times q$ matrix $\left(f_{i j}\right)_{1 \leqq i \leqq p ; 1 \leqq j \leqq q}$. Then $F:[\mathscr{E}(\Omega)]^{q} \rightarrow[\mathscr{E}(\Omega)]^{p}$ and $\operatorname{im}(F)=M$. In [2, pp. 21-25], Malgrange shows that $M=\operatorname{im}(F)$ is closed in $[\mathscr{E}(\Omega)]^{p}$ if each $f_{i j}$ is real analytic on $\Omega$. A zero of a function is said to be a zero of finite order if some derivative of the function fails to vanish there. Our main result is

THEOREM 1. Suppose $F=\left(f_{i j}\right)_{1 \leqq i \leqq p ; 1 \leqq j \leqq q}, f_{i j} \in \mathscr{E}(\Omega)$, and let $r=$ $\max \{\operatorname{rank}(F(x)): x \in \Omega\}$. For $\Omega \subset \mathscr{R}^{n}$, if the finitely generated submodule $\operatorname{im}(F)$ is closed in $[\mathscr{E}(\Omega)]^{p}$, then for every $x \in \Omega$ with $\operatorname{rank}(F(x))<r$ there exists an $r \times r$ submatrix $A$ of $F$ such that $x$ is a zero of finite order of $\operatorname{det}(A)$. For $\Omega \subset \mathscr{R}^{1}$, the converse also holds.

For $\Omega \subset \mathscr{R}^{n}, n>1$, the converse fails to hold [1, p. 89]. For $\Omega \subset \mathscr{R}^{1}$, the fact that the zeros of finite order condition is sufficient follows from Malgrange's characterization of the closure of a submodule of differentiable functions [1, Corollary 1.7, p. 25]. For $\Omega \subset \mathscr{R}^{n}$, the necessity of the zeros of finite order condition can be demonstrated in the following manner. Assuming that $\operatorname{im}(F)$ is closed in $[\mathscr{E}(\Omega)]^{p}$, we have by the closed range theorem for Frechet spaces that $\operatorname{im}\left(F^{\prime}\right)=[\operatorname{ker}(F)]^{\perp}$ where $F^{\prime}:\left[\mathscr{E}^{\prime}(\Omega)\right]^{p} \rightarrow\left[\mathscr{E}^{\prime}(\Omega)\right]^{q}$ is the transpose of $F$. Assuming that the set $Z_{\infty}$ of $x \in \Omega$ for which $x$ is a zero of infinite order of $\operatorname{det}(A)$ for every $r \times r$

AMS (MOS) subject classifications (1970). Primary 46E25, 46E40; Secondary 34A30, $35 \mathrm{G} 05,46 \mathrm{~F} 10$.

Key words and phrases. Spaces of differentiable functions, modules of differentiable functions, finitely generated submodules, spaces of distributions, systems of linear differential equations. 
submatrix $A$ of $F$ is nonempty, we find that there exists $\left(T_{1}, \ldots, T_{q}\right) \in$ $[\operatorname{ker}(F)]^{\perp}$ such that for some $j, 1 \leqq j \leqq q, \operatorname{supp}\left(T_{j}\right)=\{a\} \subset \operatorname{bd}\left(Z_{\infty}\right)$. Therefore $F^{\prime}\left(S_{1}, \ldots, S_{p}\right)=\left(T_{1}, \ldots, T_{q}\right)$ for some $\left(S_{1}, \ldots, S_{p}\right) \in\left[\mathscr{E}^{\prime}(\Omega)\right]^{p}$ which leads to a distribution equation of the form $g_{1} S_{1}+\cdots+g_{p} S_{p}=T$ where each $g_{i}$ has a zero of infinite order at $a$ and $\operatorname{supp}(T)=\{a\}$, which is impossible. A complete proof of Theorem 1 will appear elsewhere.

3. Applications. For $F=\left(f_{i j}\right)_{1 \leqq i \leqq p ; 1 \leqq j \leqq q}, f_{i j} \in \mathscr{E}(\Omega)$, define $F:\left[\mathscr{D}^{\prime}(\Omega)^{q} \rightarrow\right.$ $\left[\mathscr{D}^{\prime}(\Omega)\right]^{p}$ by

$$
F\left(S_{1}, \ldots, S_{q}\right)=\left(\sum_{j=1}^{q} f_{1 j} S_{j}, \ldots, \sum_{j=1}^{q} f_{p j} S_{j}\right)
$$

and let $F^{\prime}:[\mathscr{D}(\Omega)]^{p} \rightarrow[\mathscr{D}(\Omega)]^{q}$ be the transpose of $F$.

Suppose $P_{1}, \ldots, P_{q}$ are constant coefficient linear differential operators and consider the system of variable coefficient linear differential equations

$$
f_{i 1} P_{1} S_{1}+\cdots+f_{i q} P_{q} S_{q}=T_{i}, \quad 1 \leqq i \leqq p,
$$

where each $T_{i} \in \mathscr{D}^{\prime}(\Omega)$. In order that there exist a solution $\left(S_{1}, \ldots, S_{q}\right) \in$ $\left[\mathscr{D}^{\prime}(\Omega)\right]^{q}$ to $(1)$, it is necessary that $\left(T_{1}, \ldots, T_{p}\right) \in\left[\operatorname{ker}\left(F^{\prime}\right)\right]^{\perp}$ since $\operatorname{im}(F) \subset\left[\operatorname{ker}\left(F^{\prime}\right)\right]^{\perp}$. Equivalently, it is necessary that every "relation" between the rows of $\left(f_{i j}\right)_{1 \leqq i \leqq p ; 1 \leqq j \leqq q}$ be a "relation" between $\left(T_{1}, \ldots, T_{p}\right)$, that is, if $\psi_{1} f_{1}+\cdots+\psi_{p} f_{p}=(0, \ldots, 0)$ where $f_{i}=\left(f_{i 1}, \ldots, f_{i q}\right)$ and $\psi_{i} \in \mathscr{D}(\Omega)$ for $1 \leqq i \leqq p$, then $\psi_{1} T_{1}+\cdots+\psi_{p} T_{p}=0$. When is this condition also sufficient? A partition of unity argument, the closed range theorem, and Theorem 1 give

THEOREM 2. Suppose $F=\left(f_{i j}\right)_{1 \leqq i \leqq p ; 1 \leqq j \leqq q}, f_{i j} \in \mathscr{E}(\Omega)$, and let $r=$ $\max \{\operatorname{rank}(F(x)): x \in \Omega\}$. For $\Omega \subset \mathscr{R}^{n}$, if there exists a solution $\left(S_{1}, \ldots, S_{q}\right) \in$ $\left[\mathscr{D}^{\prime}(\Omega)\right]^{q}$ to $(1)$ for every $\left(T_{1}, \ldots, T_{p}\right) \in\left[\operatorname{ker}\left(F^{\prime}\right)\right]^{\perp}$, then for every $x \in \Omega$ with $\operatorname{rank}(F(x))<r$ there exists an $r \times r$ submatrix $A$ of $F$ such that $x$ is a zero of finite order of $\operatorname{det}(\mathrm{A})$. For $\Omega \subset \mathscr{R}^{1}$, the converse also holds.

When can (1) be solved for every $\left(T_{1}, \ldots, T_{p}\right) \in\left[\mathscr{D}^{\prime}(\Omega)\right]^{p}$ ? Using the fact that $F^{\prime}:[\mathscr{D}(\Omega)]^{p} \rightarrow[\mathscr{D}(\Omega)]^{q}$ is one-to-one if and only if the set of $x \in \Omega$ for which $\operatorname{rank}(F(x))=p$ is dense in $\Omega$, it is easy to see that the analog of Theorem 2 in this case involves the following condition: For every $x \in \Omega$ with $\operatorname{rank}(F(x))<p$ there exists a $p \times p$ submatrix $A$ of $F$ such that $x$ is a zero of finite order of $\operatorname{det}(A)$.

Theorem 1 can also be applied to systems of variable coefficient linear differential equations of the form

$$
f_{i 1} P_{1} g_{1}+\cdots+f_{i q} P_{q} g_{q}=h_{i}, \quad 1 \leqq i \leqq p,
$$

where each $f_{i j} \in \mathscr{E}(\Omega)$, each $P_{j}$ is a constant coefficient linear differential 
operator, and each $h_{i} \in \mathscr{E}(\Omega)$. In order that there exist a solution $\left(g_{1}, \ldots, g_{q}\right) \in[\mathscr{E}(\Omega)]^{q}$ to $(2)$, it is necessary that $\left(h_{1}, \ldots, h_{p}\right)$ be "pointwise"

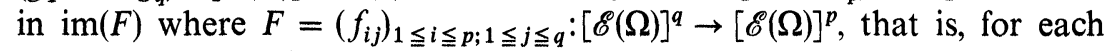
$x \in \Omega, T_{x}\left(h_{1}, \ldots, h_{p}\right) \in T_{x}(\mathrm{im}(F))$ where $T_{x}$ is the natural mapping of $[\mathscr{E}(\Omega)]^{p}$ onto $[\mathscr{E}(\Omega)]^{p} /\left[J_{x}\right]^{p}$ and $J_{x}$ is the ideal in $\mathscr{E}(\Omega)$ consisting of all functions in $\mathscr{E}(\Omega)$ which vanish at $x$ together with all derivatives. When is this condition also sufficient? Malgrange's characterization of the closure of a submodule of differentiable functions and Theorem 1 give

Theorem 3. Suppose $F=\left(f_{i j}\right)_{1 \leqq i \leqq p ; 1 \leqq j \leqq q}, f_{i j} \in \mathscr{E}(\Omega)$, and let $r=$ $\max \{\operatorname{rank}(F(x)): x \in \Omega\}$. For $\Omega \subset \mathscr{R}^{n}$, if there exists a solution $\left(g_{1}, \ldots, g_{q}\right) \in$ $[\mathscr{E}(\Omega)]^{q}$ to (2) for every $\left(h_{1}, \ldots, h_{p}\right)$ which is pointwise in $\operatorname{im}(F)$, then for every $x \in \Omega$ with $\operatorname{rank}(F(x))<r$ there is an $r \times r$ submatrix $A$ of $F$ such that $x$ is a zero of finite order of $\operatorname{det}(A)$. For $\Omega \subset \mathscr{R}^{1}$, the converse also holds.

When can (2) be solved for every $\left(h_{1}, \ldots, h_{p}\right) \in[\mathscr{E}(\Omega)]^{p}$ ? It is easy to see (even without Theorem 3 ) that the analog of Theorem 3 in this case involves the following condition: $\operatorname{rank}(F(x))=p$ for all $x \in \Omega$. And assuming that $\Omega \subset \mathscr{R}^{n}$ is $P_{j}$-convex for $1 \leqq j \leqq q$ (which is always the case for $\Omega \subset \mathscr{R}^{1}$ ), this condition is both necessary and sufficient.

\section{BIBLIOGRAPHY}

1. B. Malgrange, Ideals of differentiable functions, Tata Inst. Fund. Res. Studies in Math., no. 3, Tata Institute of Fundamental Research, Bombay; Oxford Univ. Press, London, 1967. MR 35 \# 3446.

2. 1 Division des distributions, Séminaire Schwartz 1959/60, Faculté des Sci., Paris, 1960, pp. 21-25. MR 23 \# A2275.

3. B. Roth, Finitely generated submodules of differentiable functions, Proc. Amer. Math. Soc. 34 (1972), 433-439.

Department of Mathematics, University of Wyoming, LaRAmie, Wyoming 82070 